\title{
Self-organization Scheme of WSNs with Mobile Sensors and Mobile Multiple Sinks for Big Data Computing
}

\author{
Ahreum Shin ${ }^{1}$, Intae Ryoo ${ }^{2+4}$, Seokhoon Kim ${ }^{3 \text { *t }}$ \\ ${ }^{1}$ Dept. Computer Science and Engineering, Kyung Hee University \\ Seoul, Korea \\ [e-mail: ashin@khu.ac.kr] \\ ${ }^{2}$ Dept. Computer Science and Engineering, Kyung Hee University \\ Seoul, Korea \\ [e-mail: itryoo@khu.ac.kr \\ ${ }^{3}$ Dept. Computer Software Engineering, Soonchunhyang University \\ Asan, Korea \\ *Corresponding author: Intae Ryoo and Seokhoon Kim
}

Received September 30, 2019; revised December 1, 2019; accepted December 12, 2019; published March 31, 2020

\begin{abstract}
With the advent of IoT technology and Big Data computing, the importance of WSNs (Wireless Sensor Networks) has been on the rise. For energy-efficient and collection-efficient delivery of any sensed data, lots of novel wireless medium access control (MAC) protocols have been proposed and these MAC schemes are the basis of many IoT systems that leads the upcoming fourth industrial revolution. WSNs play a very important role in collecting Big Data from various IoT sensors. Also, due to the limited amount of battery driving the sensors, energy-saving MAC technologies have been recently studied. In addition, as new IoT technologies for Big Data computing emerge to meet different needs, both sensors and sinks need to be mobile. To guarantee stability of WSNs with dynamic topologies as well as frequent physical changes, the existing MAC schemes must be tuned for better adapting to the new WSN environment which includes energy-efficiency and collection-efficiency of sensors, coverage of WSNs and data collecting methods of sinks. To address these issues, in this paper, a self-organization scheme for mobile sensor networks with mobile multiple sinks has been proposed and verified to adapt both mobile sensors and multiple sinks to 3-dimensional group management MAC protocol. Performance evaluations show that the proposed scheme outperforms the previous schemes in terms of the various usage cases. Therefore, the proposed self-organization scheme might be adaptable for various computing and networking environments with big data.
\end{abstract}

Keywords: WSN (Wireless Sensor Network), multi-sink, IoT (Internet of Things), Mobile Sensor Network, Big Data Computing

This research was supported by Korea Electric Power Corporation (Grant number : R18XA02), and this work was supported by the Soonchunhyang University Research Fund.

$\dagger$ These authors have contributed equally to this work. 


\section{Introduction}

The world is changing rapidly with the fourth industrial revolution and one of the keywords is 'IoT (Internet of Things)'. The IoT technology makes the connections between the objects. The data are transmitted and gathered through the internet. By big data technologies, the huge data are processed to make useful information for not only daily life [1][2] but also industry and general society [3].

With the advent of IoT technology, the importance of WSN (Wireless Sensor Network) has been on the rise. Collecting data is the basic step for IoT technologies and big data technologies. For the efficient data transmission, a lot of novel MAC protocols and the schemes are proposed and these are the foundation for the IoT.

The initial MAC protocols and schemes are designed to collect the data based on the static location. Sensors, as well as sinks, are deployed at a certain position and all the changes in topology are the failures caused by running out of battery or physical problem.

However, sensors become to be able to move recently. Even some studies are considering the mobility of the sink. Vehicles, drones, mobile phones, and many other things can be an example of mobile sensors and mobile sinks. For example, drones and robots can collect data where the nuclear disaster occurred. Also, it is possible to search survivors using drones when an accident happens at sea or on a mountain. After collecting data, the data can be accumulated and make useful information such as where the accidents occurred frequently and etc.

Yet, because the previous studies on MAC protocols are based on stationary nodes, they can not be used in the dynamic topology. Since the MAC protocols and schemes are not designed to adjust to the dynamic topology, a lot of failures happen during the transmission. In other words, the previous MAC protocols are not or rarely check the neighbors' existence. Thus, the sensor cannot recognize that their neighbors are not there even after the neighbors have moved to other locations. The sensor nodes only try to communicate with other nodes repeatedly because they cannot recognize changes. This eventually causes the waste of energy. Also, it makes the congestion as they keep trying to communicate. It eventually affects the stability of the network. Therefore, novel MAC protocols and schemes are proposed for the new WSN environment which contains mobility.

Additionally, these environments have height. Of course, the real world is a 3-dimensional environment, but the degree is considerably different compared to the previous environment. The previous environment is almost flat that it is possible to ignore the difference of height. Therefore, the height becomes an important factor too.

To take these factors into consideration, 3-dimensional group management MAC (3D-GM-MAC) protocol [4] has been proposed. However, it still has problems caused by network expansion. If the size of a network is very large, it may have longer delay, bottleneck phenomenon or data loss which impede the collection-efficiency. With the advance in technology, the data of ozone can be collected on a national scale [5]. It is possible to gather the data where nuclear disasters occur [6]. Also, the target field can be undersea to research for marine lives [7]. Coupled with big data technologies, the data enable analyzing the global ozone map to measure the seriousness of ozone layer and planning how to prevent the ozone layer disapearance. They also enable tracking the marine lives so that people can understand the marine ecosystem. Thus, the problems caused by the huge size of networks should be resolved. 
Multiple sinks can resolve this problem by enabling the network expansion. Multiple sinks can lessen the number of sensor nodes that each sink have to cover. Despite of the cost for installing more gateways, multiple sinks can collect data with less delay, less bottleneck phenomenon and less data loss. In other words, they can improve the collection-efficiency compared to the single sink.

For example, electricity transmission and distribution networks are large enough not to be covered by one gateway. The multiple sinks enables easier maintanance of the networks. Of course, except the examples above, any networks large enough to apply multiple sinks can be the target fields.

In this paper, to adapt the mobile multi-sink to the 3-dimensional group management MAC protocol, a self-organization scheme for mobile sensor networks with mobile multi-sink is proposed. The processes of initial setting and resetting group number are proposed. Also with the periodic initial setting, it prevents from propagating the wrong information of the nodes such as group number.

\section{Related Work}

WSN is a foundation of IoT system. WSN makes the IoT system possible to collect the data from various sensors. Also, the MAC protocols used in WSN have proposed recently. However, because of the advance in technology, the sensors become to have mobility. The sensor nodes in the WSN become to move and the name has been changed to WMSN (Wireless Mobile Sensor Network). Coupled with this change, the MAC protocols also have changed to adjust the dynamic topology.

\subsection{CSMA/CA (Carrier Sense Multiple Access / Collision Avoidance)}

In wireless networks, a node which wants to participate in the communication checks the medium before transmitting its data. It waits for a certain time period to make sure that the medium is free. And then, it generates a random value and waits for the amount of generated random value. If the medium is free even after all the waitings, the node sends RTS (Request to Send) packet to the node which is the destination of its data. If the node gets the CTS (Clear to Send) packet from the destination node, it starts to transmit its data. If not, it retransmits the RTS packet by the predetermined number of times. If it fails to get CTS packet, even after sending all the RTS packets, it waits again for the certain time period [8].

\subsection{S-MAC (Sensor-MAC)}

$\mathrm{S}-\mathrm{MAC}$ is the most typical type of contention-based MAC protocols. The biggest problem for sensor nodes in contention-based MAC protocol is the idle listening state. Because sensor nodes usually have limited energy, reducing energy consumption is important [9][10].

Therefore, in S-MAC, each node periodically goes into sleeping state to reduce the time for idle listening. Following the CSMA/CA, the nodes transmit their data during the active time. After the active time, the node goes into sleeping state. The active and sleep time is predetermined. Fig. 1 shows the basic scheme of S-MAC. 


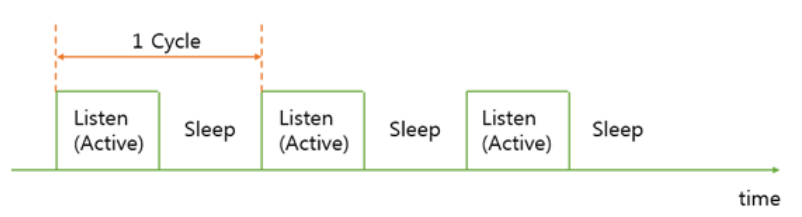

Fig. 1. Basic scheme of S-MAC with periodical listen and sleep

\subsection{T-MAC (Timeout-MAC)}

T-MAC is an enhanced version of S-MAC. The sensor nodes dynamically end the listening period. They end the listening state after the end of transmitting data. Fig. 2 shows the basic scheme of T-MAC. Because the node sleeps after transmitting data, the time for active state reduces and it makes the energy saving compared to the S-MAC [11].

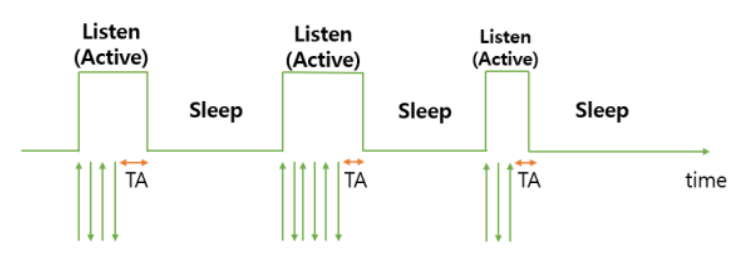

Fig. 2. T-MAC duty cycle

\subsection{E'-MAC (Energy-Efficient MAC)}

$E^{2}$-MAC adopts the idea of buffer threshold. Each sensor node has its own buffer threshold. When the amount of data in the sensor node exceeds the buffer threshold set previously, it transmits the data. With the buffer threshold, the efficiency of energy increases compared to the T-MAC [11][12].

\subsection{M-MAC (Mobility-adaptive MAC)}

M-MAC is a schedule-based MAC protocol [13][14] based on the design of TRAMA[15]. $\mathrm{M}-\mathrm{MAC}$ breaks the time into rounds and each round has $\mathrm{k}$ frames. The frame has two fields; scheduled access field and random access field.

Each node predicts its future mobility using the AR-1 mobility estimation model. While the scheduled access and random access field have fixed length in TRAMA, the length of each access field varies depending on the frame size that the cluster head has calculated by using the mobility prediction. Fig. 3 shows the frame structure of M-MAC compared to the TRAMA.

Fixed Frame Time (TRAMA)

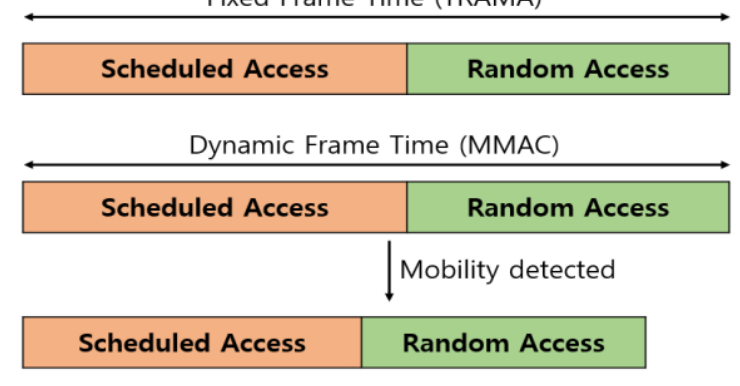

Fig. 3. Frame structure of M-MAC 


\subsection{M-TDMA (Mobility-aware TDMA based MAC)}

M-TDMA is founded on TDMA (Time Division Multiple Access). It has been proposed to adapt to the network composed with mobile sensor nodes [16].

First, it divides the network into non-overlapping clusters by FLOC algorithm. Each cluster has a cluster head. Each node has one assigned slot. Some slots are shared across the clusters for mobile nodes and some are not assigned to other nodes for future allocation. These free slots are for the nodes which will enter this cluster in the future or retransmissions.

Another characteristic is that it halves the slot when it only have one slot left to assign. Because of this characteristic, it can still have a time slot to assign in the future even though the length is shortened.

\subsection{MS-MAC (Mobility-aware MAC protocol for sensor networks)}

MS-MAC is an extended version of S-MAC which supports the mobility of sensor nodes. It has sleep and listen duty cycles and periodic synchronization. In MS-MAC, the period of synchronization varies with the degree of mobility. If the sensor node has small mobility, the period gets longer. On the other hand, if the mobility gets bigger, the period of synchronization gets shorter. This is because the topology has more changes as the nodes get stronger mobility. Therefore the more frequent adjusting is needed [17].

\subsection{GM-MAC (Group Management MAC)}

GM-MAC is based on the T-MAC. It is proposed for the network with mobile sensor nodes with a stationary sink. The basic principle of GM-MAC is grouping the sensor nodes by the distance from the sink [18]. Data are relayed to the upper group node until they reach to the sink. The process is divided into two, which are the initial setting and resetting group number. GM-MAC uses the preamble in recognizing the neighbors and setting the group number. Fig. 4 shows the basic scheme of GM-MAC after the initial setting .

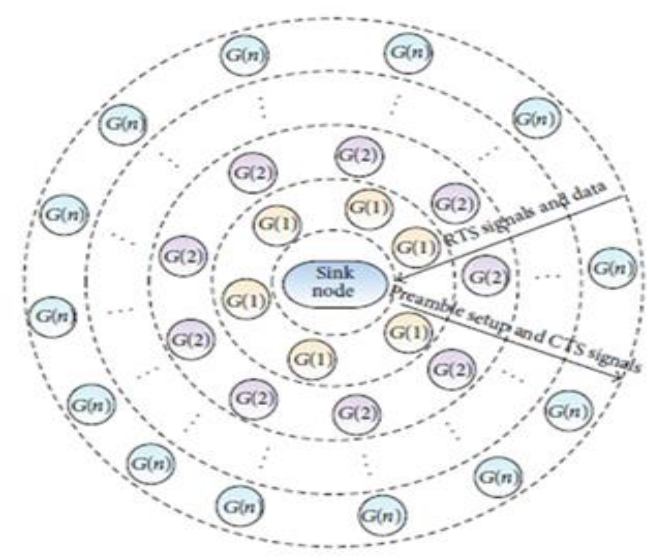

Fig. 4. Basic scheme of GM-MAC

\section{3-Dimensional Group Management MAC}

GM-MAC is designed for the wireless mobile sensor network as mentioned at $\amalg-8$. 3D-GM-MAC (3-Dimensional Group Management MAC) is an extended version of 
GM-MAC. It extends the GM-MAC to the 3-dimensional environment. Fig. 5 shows the basic scheme of 3D-GM-MAC [7].

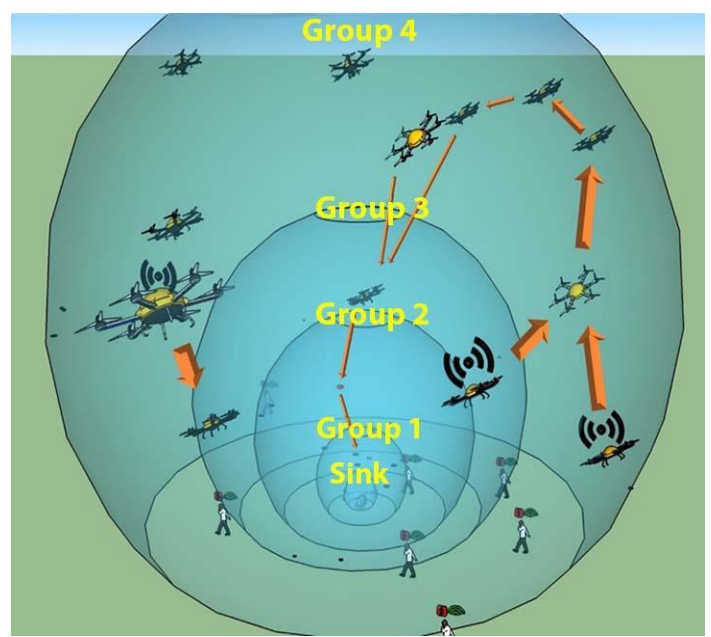

Fig. 5. Basic scheme of 3D-GM-MAC

\subsection{Initial Group ID Setting}

Initial setting is for setting the group ID of each node at the first time of communicating. Advertisement packet is used for the initial setting. It contains the group ID and the version of group ID. The version of group ID is updated every update of the group number.

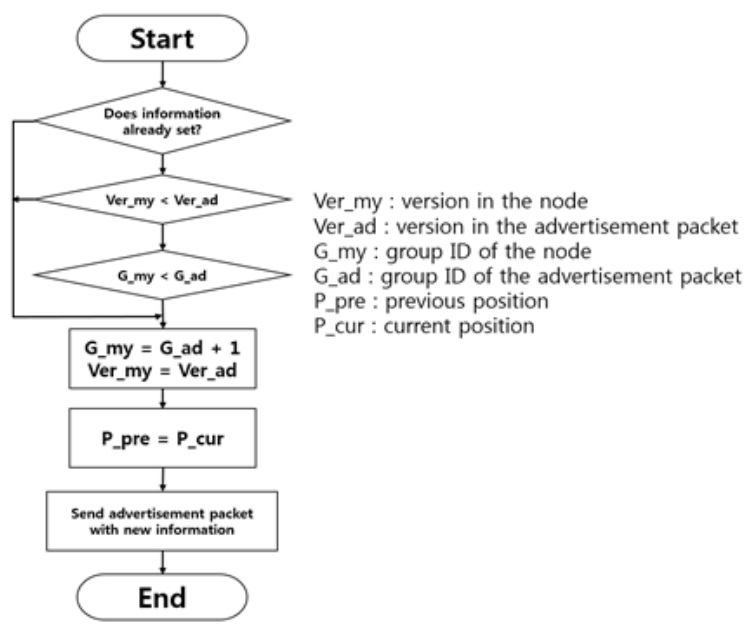

Fig. 6. Flowchart of Initial Group ID Setting

First, the sink broadcasts the advertisement packet, which contains its group ID 0 , to the neighbors. The neighbors which received the packet set their group ID as 1. After setting the information, the nodes with group ID 1 send their advertisement packet to their neighbors. Of course, the parent node can receive the advertisement packet from their child node. In this case, the parent node discards the advertisement packet and does not generate another advertisement packet. By propagating of advertisement packet from the sink, the initial setting is finished. 
Also, every time the node receives the advertisement packet, it compares the version of group ID. The node only updates their information when the advertisement packet has newer information. Fig. 6 shows the flowchart of Initial Group ID Setting and Fig. 7 shows the result of initial setting.

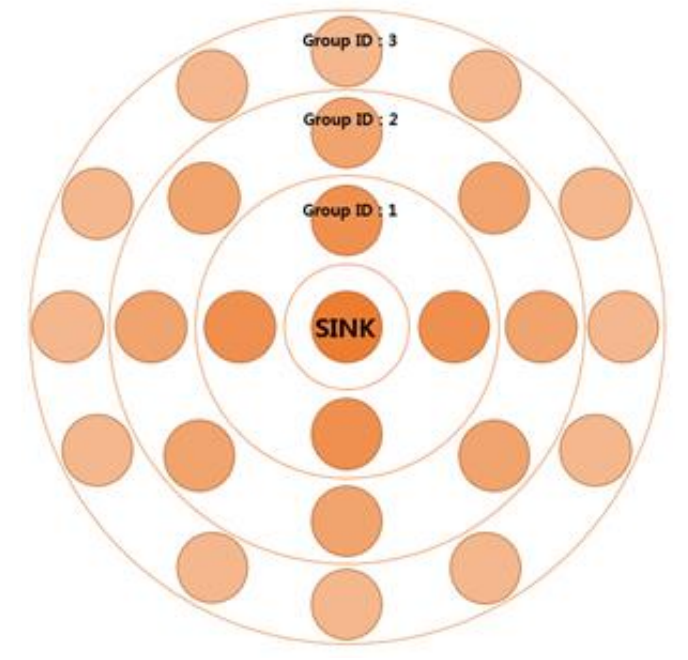

Fig. 7. Result of initial setting in 3D-GM-MAC

\subsection{Update of the Group ID}

In 3D-GM-MAC, there are two circumstances that a node updates its group ID. One is when the moving distance of the node exceeds the maximum communication range of the node. The other is when the node cannot transmit its data to the upper group node.

Under these two circumstances which are mentioned above, the node updates its group ID. For the update of group ID, the node sends hello packet to the neighbors. The neighbors which received an answer hello packet back with the reply packet including their group ID. After getting all the reply packets, the node starts to calculate the new group ID.

If the node receives a reply packet from the sink, it immediately sets its group ID as 1 . Otherwise, it compares between the $\min (\mathrm{Gn})$, which means the gsmallest group ID among the reply packets, and the result of GroupNum(listreply). If the new group ID is smaller than the smallest group ID, it is impossible to set its parent node and transmit data. So, if the result of GroupNum(listreply) is smaller than the $\min (\mathrm{Gn})$, the node sets its group ID as $\min (\mathrm{Gn})+1$ to make it possible to set the parent node to transmit its data. Or it configures its group ID as the result of GroupNum(listreply).

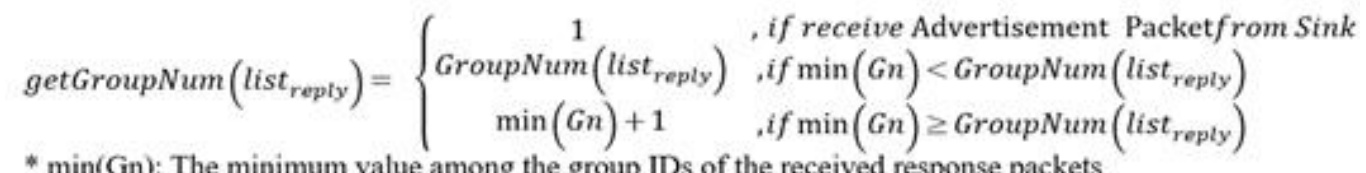

* $\min (\mathrm{Gn}):$ The minimum value among the group IDs of the received response packets

The GroupNum(listreply) is a formula to calculate a new group ID, which is considering the group weight and the moving distance. Because the volumes or the areas that each group takes varies depending on the group ID, the group weight is considered. Also, if a node is moving fast, it is hard to maintain its information. For that, the formula has the multiplying (1-movingDistance/maxDistance) to put less reliability to the information of the node which is moving faster. 


$$
\operatorname{GroupNum}\left(\text { list }_{\text {reply }}\right)=\operatorname{round}\left(\frac{\sum\left(G n *\left(1-\frac{\text { movingDistance }}{\text { maxDistance }}\right) * \operatorname{groupWeight}(G n)\right)}{\Sigma\left(\left(1-\frac{\text { movingDistance }}{\text { maxDistance }}\right) * \operatorname{groupWeight}(G n)\right)}\right)
$$

Stochastically, it is likely to find a node with a bigger group number rather than one with a smaller group number. To resolve the unbalanced number of the nodes, group weight should be considered. The ratio is related to the areas or the volumes that a group number takes. Considering the idea, the formula for group weight has drawn.

For example, in the case of 2-dimensional environment, group number X occupies the area equal to the area of a circle with a radius of $X$ minus the area of a circle with a radius of $X-1$. Therefore, the area that group number $\mathrm{X}$ takes is calculated as below.

$$
\operatorname{area}(X)=X^{2}-(X-1)^{2}=X^{2}-X^{2}+2 X-1=2 X-1
$$

The expression for 3-dementional environment is derived in the same way. Thus, the formula for calculating the group weight is as below.

$$
\text { groupWeight }(G n)= \begin{cases}\frac{1}{2 * G n-1} & \text {, if the IoT systems is } 2-D \\ \frac{1}{3 *(G n-1) * G n+1} & \text {, if the IoT system is } 3-D\end{cases}
$$

However, not every node succeeds to set its new group number. It may not receive any reply packet. This may be due to some error during the transmission or simply because the node is in an isolated area. In this case, the node remains with the previous group number. Accordingly, the node repeats the same process until it sets a new group number and communicates with other nodes.

\subsection{Buffer Threshold}

In 3D-GM-MAC, the bigger the group number is, the bigger the number of the nodes in that group. Therefore, if every node has same buffer threshold, the bottleneck phenomenon or data loss can occur around the sink. Fig. 8 describes when data loss occurs when 3D-GM-MAC is applied to a network without bufferthreshold concept.

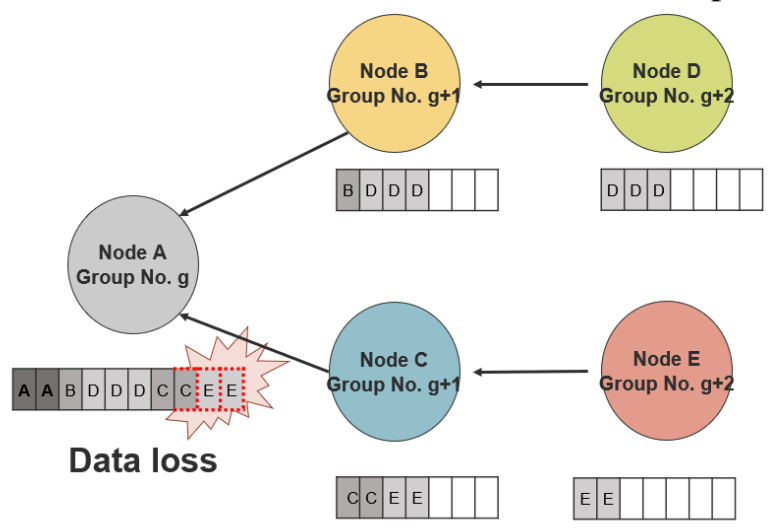

Fig. 8. Data loss when 3D-GM-MAC is applied to a network without bufferthreshold concept 
To prevent this from happening, the buffer threshold varies depends on the group ID. If a node is located at closer to the sink, it configures the buffer threshold bigger. The ratio of increase for the increasing the group ID depends on the volume or the area the group takes.

The expression (5) is for calculating the buffer threshold. It is derived from the same concept used in calculating group weight in 3.2. Of course, the buffer threshold changes when the group number changes.

$$
\text { bufferThreshold }(G n)= \begin{cases}\frac{B_{\max }}{2 * G n-1} & \text {, if the WSN is 2-D } \\ \frac{B_{\max }}{3 * G n(G n-1)+1} & \text {, if the WSN is 3-D }\end{cases}
$$

$G_{n}:$ Group Number

$B_{\max }:$ buffer size of all nodes

\section{Multi-Sink with GM-MAC}

\subsection{Necessity of Multi-sink}

In GM-MAC and 3D-GM-MAC, it is possible to have bottleneck phenomenon or data loss, especially around the sink. This is because of the characteristic of GM-MAC and 3D-GM-MAC, which is transmitting the data from the lower group nodes to the higher group nodes by relaying. Therefore, the larger the field where the MAC protocol operates is, the more the nodes are and the higher the possibility is to experience the bottleneck phenomenon or data loss.

Additionally, as a network expands, the group number increases continuously and the buffer threshold decreases. Eventually, the buffer threshold reaches to a very small value less than one data packet. Let the group number at the time is $\mathrm{N}$. The nodes with group numbers bigger than $\mathrm{N}$ will transmit the data as soon as they collect the data. That makes the concept of buffer threshold meaningless.

Also, in the perspective of energy consumption, as the size of a network gets larger, the average energy to transmit data to the sink will increase. If a node is deployed and has group number $\mathrm{M}$, data collected by the node should be transmitted $\mathrm{M}$ times to reach the sink. The delay becomes longer because it takes more time to be delivered for data from a node to the sink if the node is located in the lower group.

Multi-sink can work with these problems. Multi-sink reduces the burden that the single sink has. Multi-sink is needed where the target field is too large so that data are delivered too slowly or lost. It can be judged by the administrator. One condition can be when the maximum group number is bigger than $\mathrm{N}$; which makes the buffer threshold is less than one data unit. For example, electricity transmission and distribution system is large enough to adapt multiple sinks. Besides, skyscrapers, big smart factories, and any other system large enough can be target system.

\subsection{Sink-change Threshold}

Since there are multiple sinks in the topology, it is possible for sensors to change the sink where their data eventually transmitted to. It is because the sensors are mobile. As sensors are 
move around, a sensor can be more close to other sinks than the sink that the sensor already set. It is better to change the sensor's sink as the close one from the originally set one since it needs less data transmission and less energy.

The change happens around the boundary of multiple sinks. Because the mobility of a node is not linear, it is possible to be in and out of a sink area. Fig. 9 shows the frequent sink change during the node is moving around the boundary of multiple sinks.

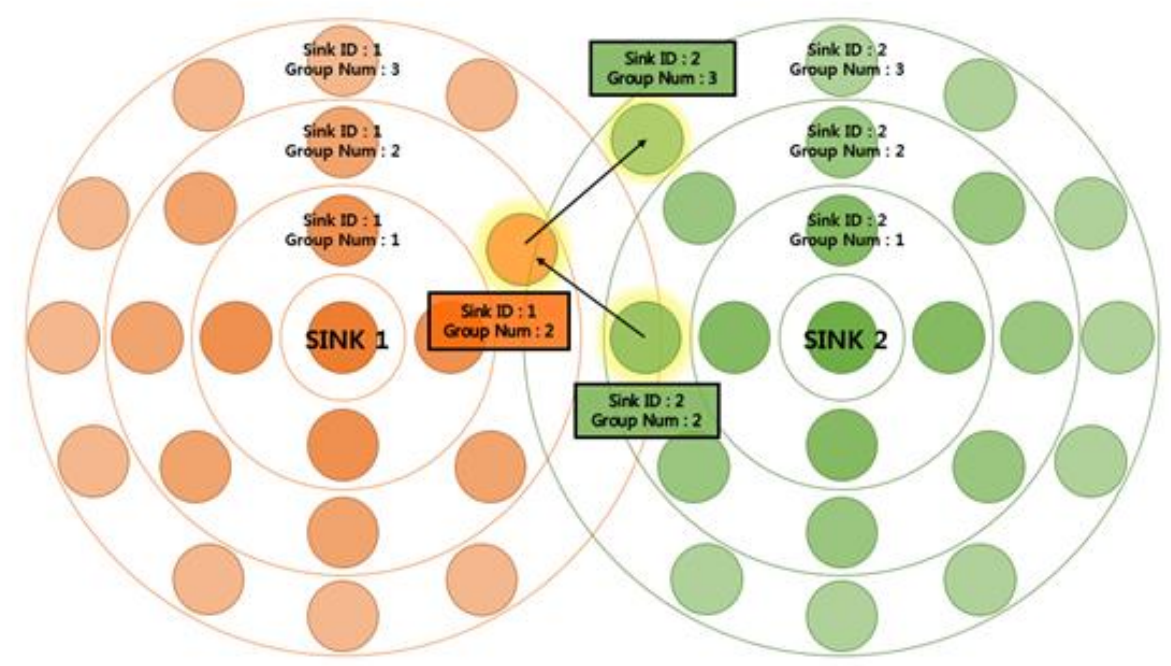

Fig. 9. Frequent sink change at the boundary of multiple sinks

This makes the sink ID of the node be changed and the frequent changes in the topology cause instability. Therefore, in this paper, the sink-change threshold $\mathrm{K}$ is used to decide whether the node passes the boundary of the multiple sinks and is included completely in the area of another sink. The specific way to adopt the sink-change threshold $\mathrm{K}$ is described in 4.4.

\subsection{Initial Setting / Periodic refreshing}

The periodic refreshing process is the same as the process of initial setting. However, they have a difference in their goal. The initial setting is done when all the nodes turn on and starts to operate first. The aim of initial setting is setting the sink ID and group number for all the sensor nodes and sinks.

The periodic refreshing is done periodically during the transmission. The period is predetermined. By the periodic refreshing, it prevents the propagating of wrong information that is given from the nodes which can not transmit its data because they already moved. If a node moves and becomes to be unable to transmit the data with its information, the information is wrong. However, it is possible to get this wrong information from its neighbor before the node updates its information to be right.

The general concept of initial setting and periodic refreshing is similar to the 3D-GM-MAC. The biggest difference is that each node has the sink ID, which indicates the sink that the node is connected to eventually. 
The sink ID of each sink has predetermined and the group numbers of sinks are all set as 0 . With this information, the nodes make advertisement packets and the each advertisement packet is transmitted to the neighbors. If a node receives an advertisement packet and the information is not set, the node sets its information based on the advertisement packet. The sink ID is configured as the one in the advertisement packet, and the group number is set by adding 1 to the group number in the advertisement packet. After that, the node makes its advertisement packet with its information and sends to the neighbors.

In the case that the information is already set when a node receives an advertisement packet, the node compares between its own information and the one in the packet. If the group number in the received advertisement packet is smaller than the one the node already has, it changes its information based on the received advertisement packet. Also, it does the process of sending its advertisement packet with updated information. Otherwise, it discards the received advertisement packet. Starting from the sinks, the advertisement packets spread out to the edge of the network. Fig. 10 shows an example of the scheme after initial setting with 2 sinks.

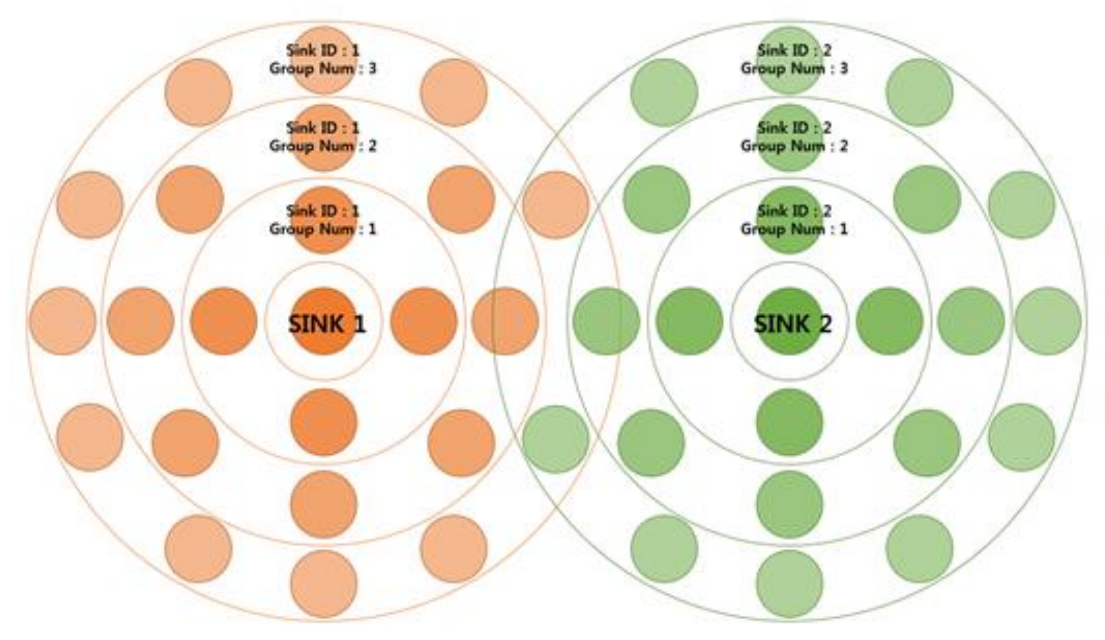

Fig. 10. An example of scheme after initial setting with 2 sinks

\subsection{Resetting Group Number}

The physical topology is continuously changing as the time goes by. This is caused by the mobilities of the sinks and sensor nodes. Therefore, it is impossible to communicate for the sensor nodes with the initial information. Fig. 11 shows a circumstance that the node A can not transmit its data anymore after all the nodes are moving. 

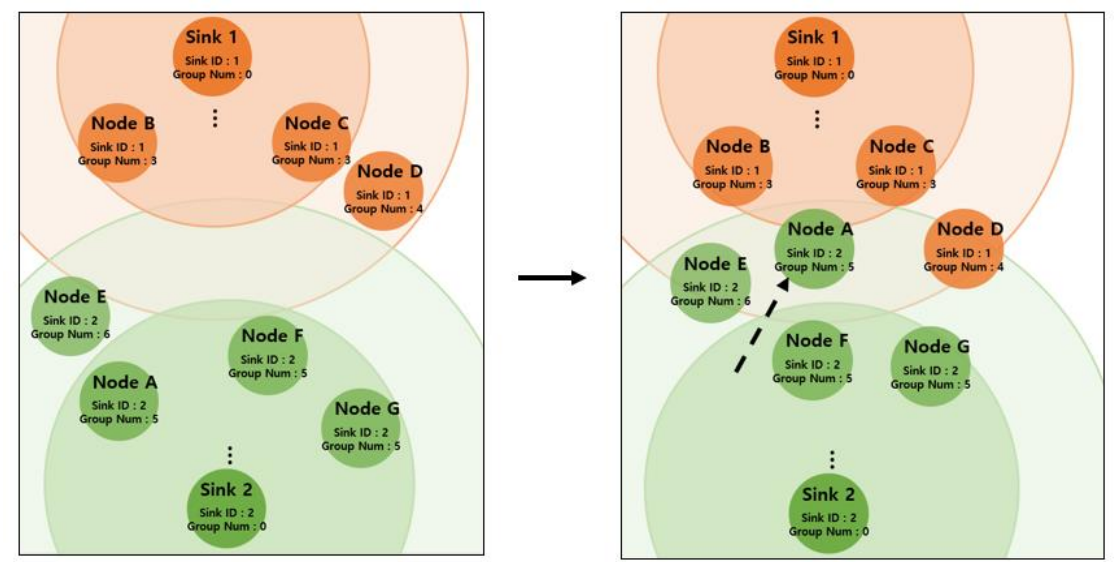

Fig. 11. A circumstance that the node A can not transmit its data after movement

If the sensor node realizes that it can't transmit its data with the information it already has, it should update its information to transmit the data. Following the original GM-MAC, a node defines the impossibility of transmission as not receiving CTS packet after sending 3 RTS packets.

If a node can't receive any CTS packet after sending 3 RTS packets, it broadcasts hello packets to its neighbors. The neighbors which received the hello packet is sent back a reply packet to the node, the source of hello packet. The reply packet contains the group number and the sink ID. The node which needs for resetting group number gathers all the reply packets and classifies the packets by their sink IDs. The group numbers are calculated by the formula in 3D-GM-MAC with the reply packets for each sink ID.

As the results, in the group numbers, group numbers for each sink are calculated. Let these group numbers are G1, G2, ..., Gs. S is the number of the sinks. Because the smallest Gn means that the node is located closest to the sink which has sink ID N. Fig. 12 shows the node which is calculating the group numbers after receiving the reply packets from its neighbors. Fig. 12 shows two sinks as an example, but if there are more sinks, the number of group numbers to be calculated increases by that amount.
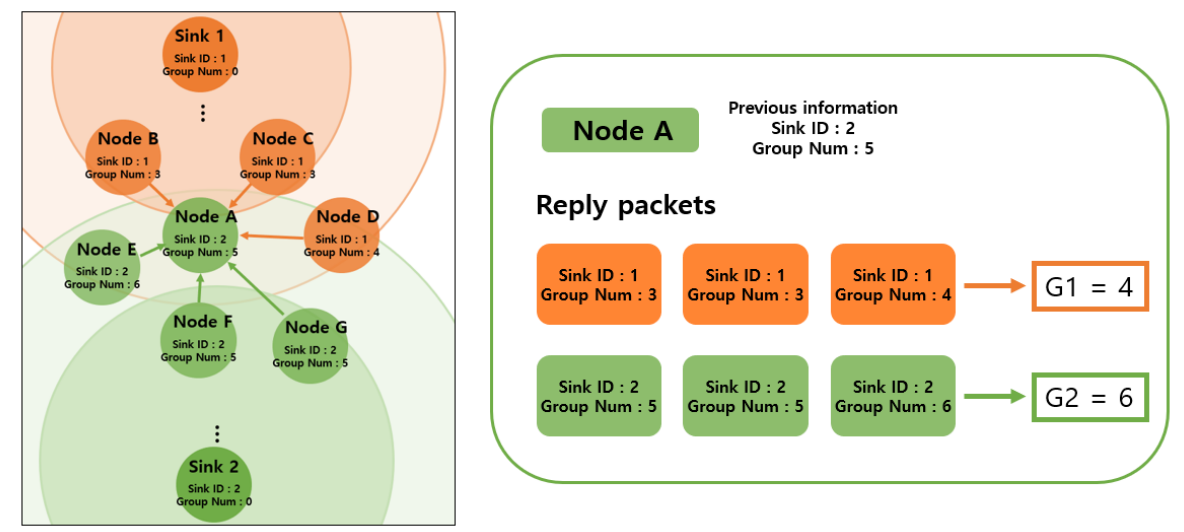

Fig. 12. An example of the scheme after calculating the group numbers based on each sink in the network with 2 sinks 
However, it is not good to choose the smallest group number Gn. If the node chooses the smallest group number Gn, the node might change its sink ID again after a while. This is the issue that mentioned in 4-2.

Therefore, the node adds the sink-change threshold $\mathrm{K}$ to the calculated group numbers except the one calculated based on the previously connected sink. The sink-change threshold $\mathrm{K}$ is globally configured so every node applies the same value as the K. Fig.13 describes that the result of adopting the sink-change threshold K to Fig. 12.
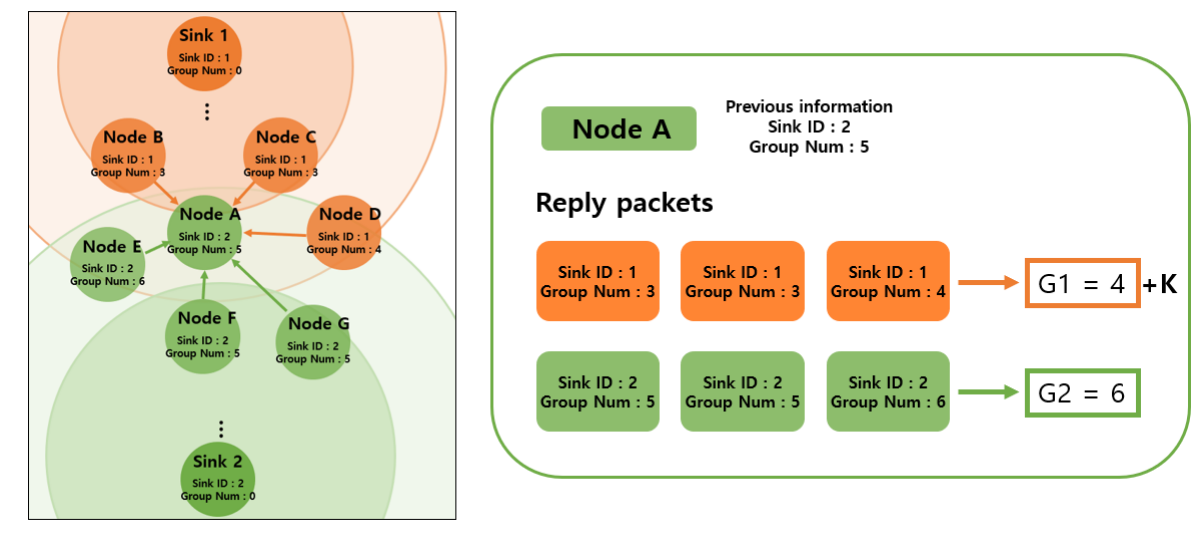

Fig. 13. The result of adopting the sink-change threshold K to Fig. 12

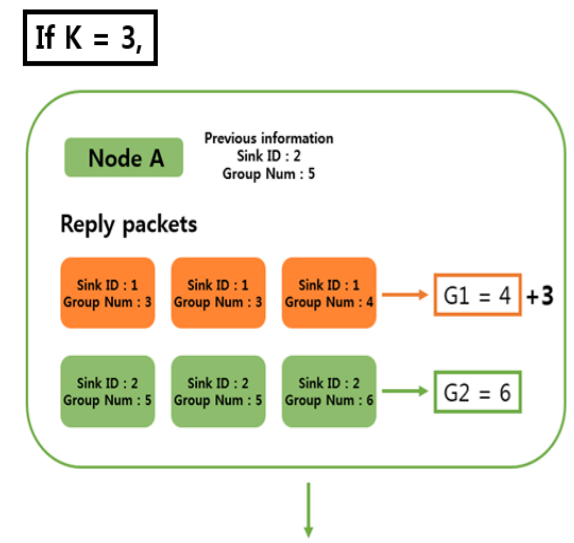

Node A
Sink ID : 2
Group Num : 6

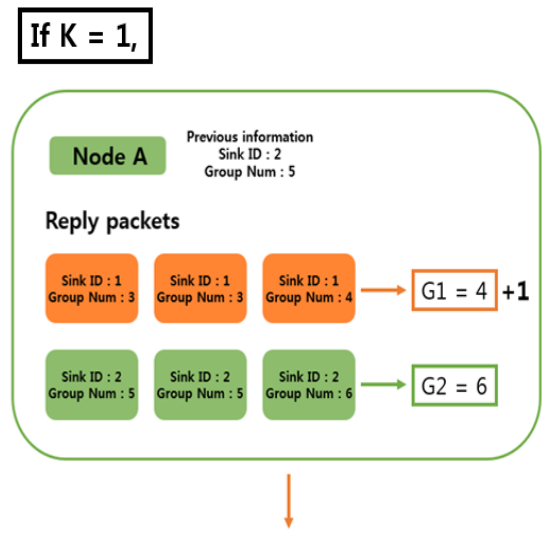

Node A $\quad \begin{gathered}\text { Sink ID : } 1 \\ \text { Group Num : } 4\end{gathered}$

Fig. 14. The results of resetting group number when the $\mathrm{K}$ is 3 (left) / The results of resetting group number when the $\mathrm{K}$ is 1 (right)

After adding $\mathrm{K}$, the rest of the process is choosing the smallest among the group numbers which are the results of adopting the sink-change threshold $\mathrm{K}$. Of course, if the smallest group number is the one added $\mathrm{K}$, the group number is set as the initial calculated group number without adding $\mathrm{K}$. The sink-change threshold $\mathrm{K}$ is only used for giving handover when the node is passing through the sinks, but not influencing the information set on the node. Fig. 14 
shows the new information for node $\mathrm{A}$ after the process of resetting group number depends on the $\mathrm{K}$. The left in Fig. 14 shows the results when the $\mathrm{K}$ is 3, and the right shows the one when the $\mathrm{K}$ is 1 .

On the other hand, the node which needs to reset its group number may not be able to receive any reply packet. In this case, as mentioned in 3.2. it repeats the same process unless it sets a new group number. However, because sensor nodes are mobile, it is likely to have neighbor nodes as sensors move around.

\section{Experimentation and Comparative Analysis}

\subsection{Simulation Environment}

This simulation is implemented by using $\mathrm{c}++$. This simulation is for monitoring the energy of each sensor node and the number of live nodes as time goes by. The Table 1 shows the specification of the simulation.

Table 1. Specification of the simulation

\begin{tabular}{|c|c|c|}
\hline Factor & Value & Remark \\
\hline Physical space & $300 \mathrm{~m} \times 300 \mathrm{~m} \times 300 \mathrm{~m}$ & 3-dimensional space, $\mathrm{x}, \mathrm{y}, \mathrm{z}$ \\
\hline Number of Sink node & 2 & \\
\hline Number of Sensor node & 100 & \\
\hline Mobility & Maximum 5 meter per minute & Sensor node movement randomly \\
\hline Initial Battery Capacity & $3000 \mathrm{~mW}$ & \\
\hline Size of Collect Data & 5bytes / 10bytes & \\
\hline Frequency of Collecting Data & Average 1 time per minute & $\begin{array}{c}\text { Data collection event by Poisson } \\
\text { distribution, } \\
\text { All nodes are collected once per } \\
\text { minute on average. }\end{array}$ \\
\hline The period of refreshing & $\begin{array}{l}\text { No periodic refreshing } \\
15 \mathrm{mins} \\
30 \mathrm{mins} \\
60 \mathrm{mins}\end{array}$ & \\
\hline $\begin{array}{c}\text { The sink-change } \\
\text { threshold } \mathrm{K}\end{array}$ & $1 / 2 / 3$ & \\
\hline Tx Energy Consumption & $0.0145 \mathrm{~mW}$ & Specification of T.I CC2420 \\
\hline Rx Energy Consumption & $0.0156 \mathrm{~mW}$ & Specification of T.I CC2420 \\
\hline $\begin{array}{c}\text { Maximum Transmission } \\
\text { Distance }\end{array}$ & $90 \mathrm{~m}$ & $\begin{array}{l}\text { Maximum transmission range of } \\
\text { CC } 2420 .\end{array}$ \\
\hline Buffer size of all nodes & 1024bytes & $\begin{array}{c}\text { Default size of Sensor node } \\
\text { Buffer }\end{array}$ \\
\hline
\end{tabular}




\subsection{Simulation Evaluation}

Fig. 15 shows the remaining energy of each node and the number of active sensor nodes. The simulation is done with $\mathrm{K}=2$ and periodic refreshing every 30 minutes. The red line above indicates the number of active sensor nodes and the rest lines show the remaining energy of each sensor nodes.

As the graph shows, all of the sensor nodes consume energy with a similar degree. That is to say, the sensor nodes stop working at a similar point. It also makes the number of active sensor nodes plummets at a certain point. This characteristic is very important. When the energy is not decreasing similarly, some sensor nodes use their battery earlier than the other nodes. It can cause the isolation of other nodes. Or, even though it does not cause the isolation of other nodes, it still has a problem of not collecting data from some places. Therefore, it is important to have the characteristic regardless of the value of $\mathrm{K}$ or the period of the refreshing process.

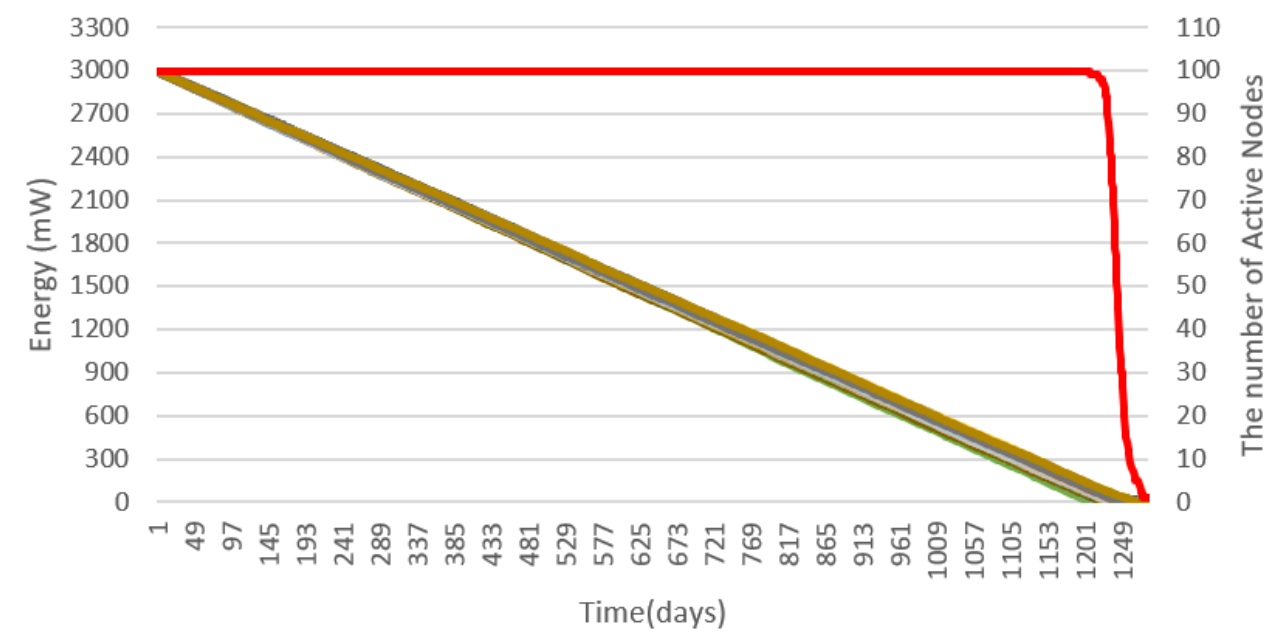

Fig. 15. Remaining energy of each node and the number of active nodes with $K=2$ and periodic refreshing every 30 mins

Fig. 16 shows the number of active nodes when $K$ is 1, 2, and 3 with refreshing process every 15 minutes. In this case, the value of $\mathrm{K}$ that makes the network lifespan longer is 2 . However, regardless of the $\mathrm{K}$, the number of active sensor nodes plummet at a certain point as mentioned before. 


\section{Refreshing process every 15 mins}

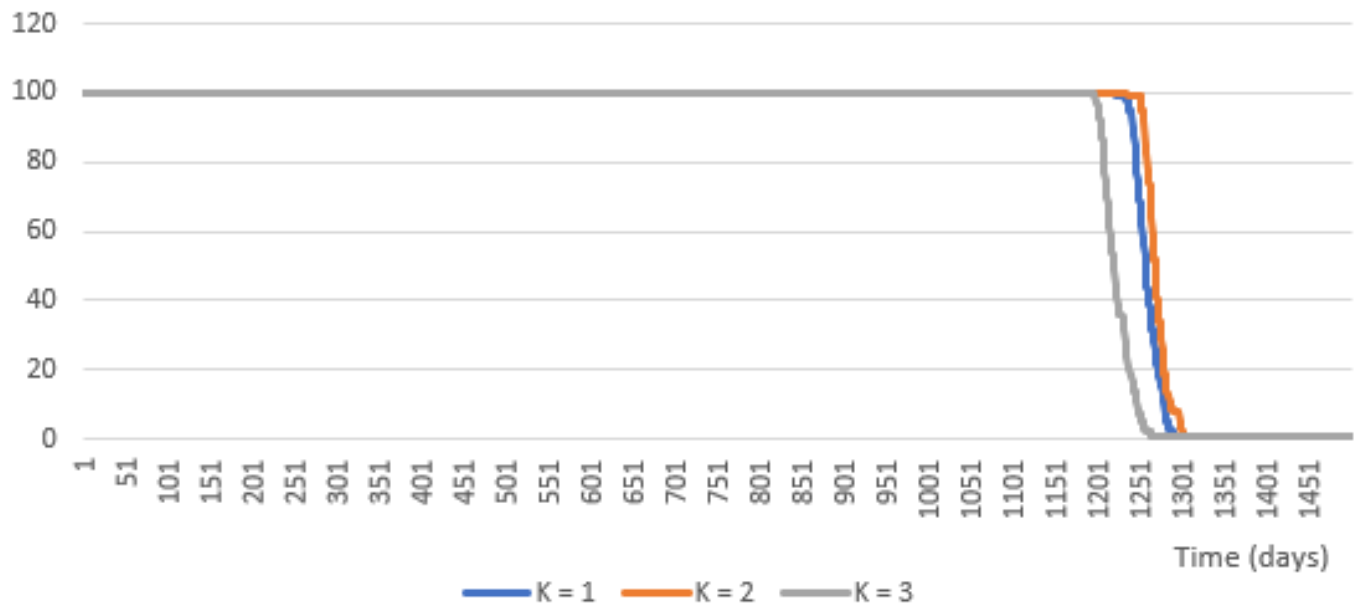

Fig. 16. The number of active nodes when $\mathrm{K}$ is 1,2 , and 3 with refreshing process every 15 mins

Fig. 17 shows the number of active nodes when $\mathrm{K}$ is 1,2 , and 3 with refreshing process every 30 minutes. Still, the lifespan of the network with $K=2$ is the longest among them. The network with $\mathrm{K}=1$ has the shortest lifespan with refreshing process every 30 minutes, while the one with $\mathrm{K}=3$ has the shortest lifespan when the refreshing process is done every 15 minutes.

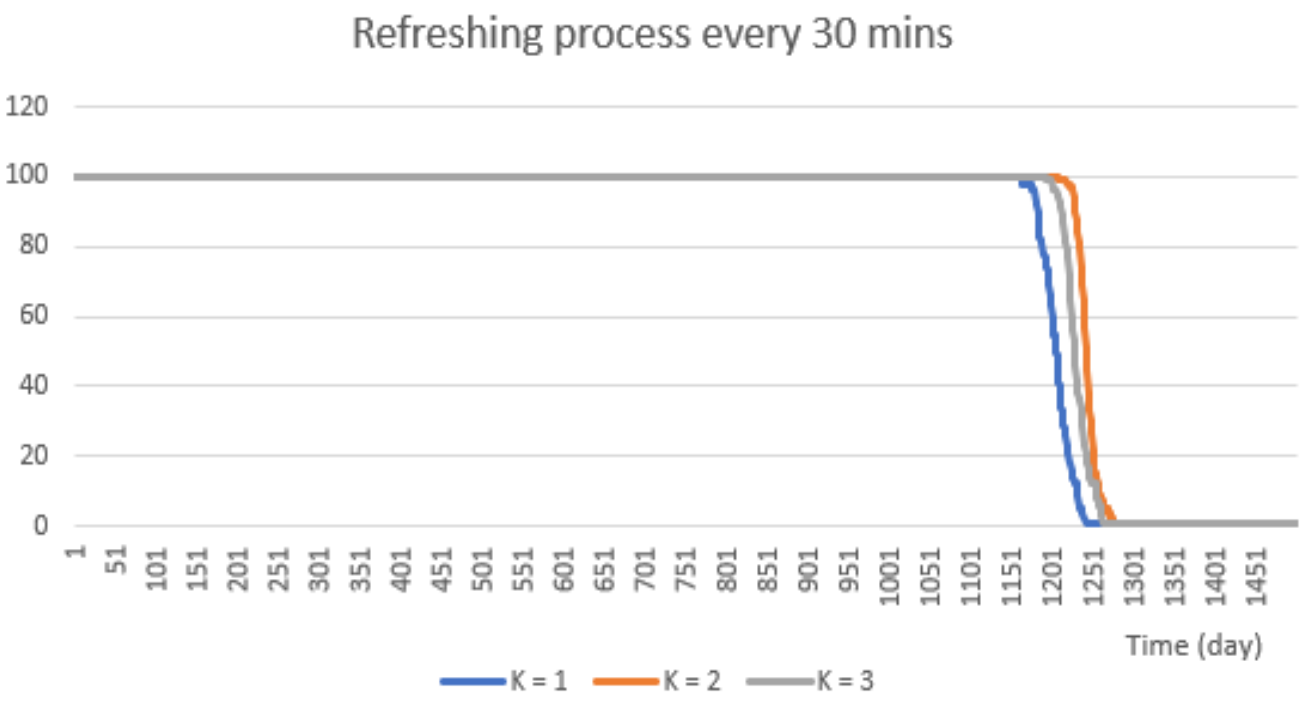

Fig. 17. The number of active nodes when $\mathrm{K}$ is 1,2 , and 3 with refreshing process every 30 mins

Lastly, Fig. 18 gives the number of active nodes when $\mathrm{K}$ is 1,2 , and 3 with refreshing process every 60 minutes. It is obvious that the network with $K=1$ is the best. Additionally, the number of active nodes decreases rapidly at a certain point regardless of the conditions. 


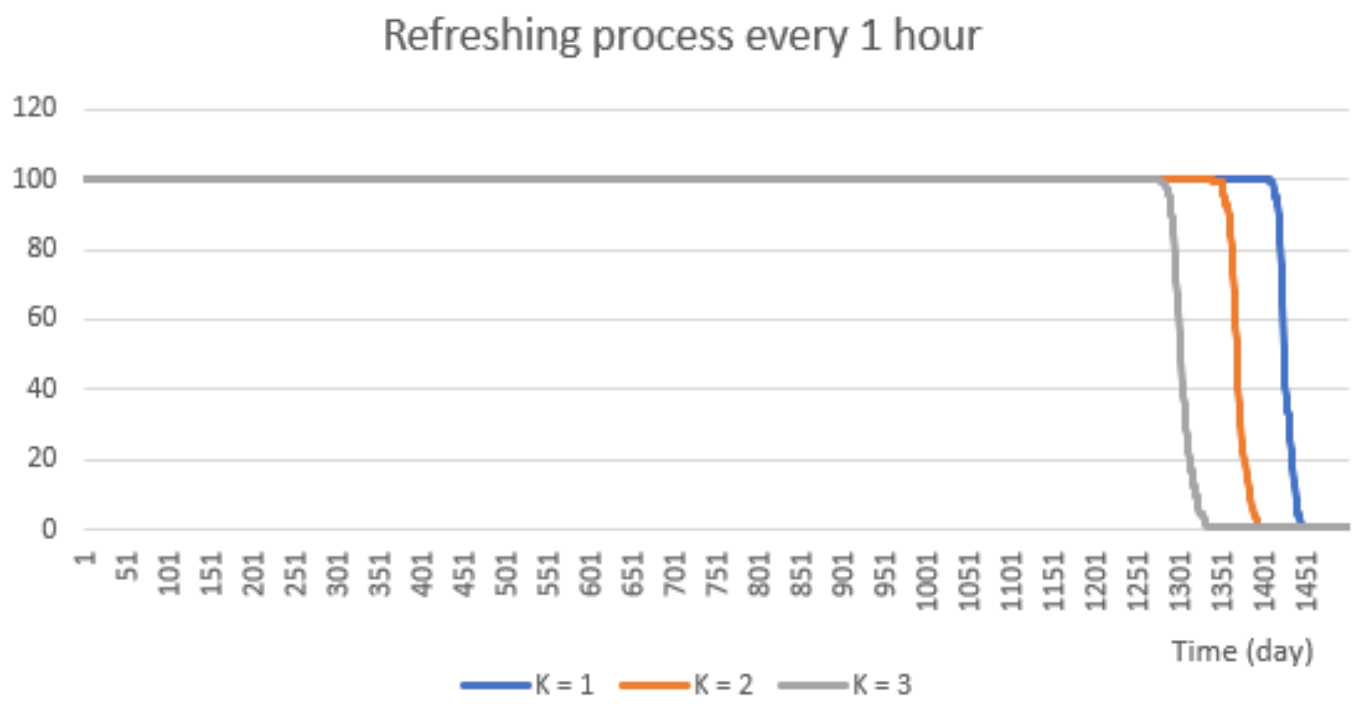

Fig. 18. The number of active nodes when $\mathrm{K}$ is 1,2 , and 3 with refreshing process every 60 mins

To sum up, the first day the battery starts to run out is all different as the Table 2 shows. By the results, the lifespan varies depending on the value of $\mathrm{K}$ and the period of refreshing process. Therefore, it is important for administrator to set appropriate sink-threshold $\mathrm{K}$ and the period of refreshing process. However, regardless of the value of $\mathrm{K}$ and the period of refreshing process, the balanced energy consumption is maintained.

Table 2. The first day when the nodes are starting to run out of their battery

\begin{tabular}{|c|c|c|c|}
\hline \multicolumn{1}{|r|}{$\begin{array}{r}\text { Sink-Threshold } \\
\begin{array}{l}\text { Refreshing } \\
\text { period }\end{array}\end{array}$} & 1 & 2 & 3 \\
\hline \hline 15 min & 1,222 & 1,235 & 1,195 \\
\hline 30 min & 1,163 & 1,207 & 1,193 \\
\hline 60 min & 1,407 & 1,340 & 1,276 \\
\hline
\end{tabular}

\section{Conclusion}

In this paper, the ways of initial setting, periodic refreshing and the resetting group number have been proposed for adapting multiple sinks to the 3D-GM-MAC. In the initial setting, the sink ID and group number are set based on their location from the sinks. It chooses the closest sink and set the information to transmit the data to the sink. Also, the periodic refreshing has the same process of the initial setting but the aim is different. It is for preventing from propagating of wrong information caused by the mobility.

Additionally, it proposes the way of resetting group numbers. When a node realizes that it can't transmit the data with its information, it tries to figure out a new sink ID and group number based on the information of its neighbors. Furthermore, it uses the sink-change 
threshold $\mathrm{K}$ for preventing a node from frequent sink change when it passes through the boundary of multiple sinks.

Finally, the results of the simulation show that the energy of each node reduces with similar degrees, regardless of the period of refreshing or the sink-change threshold K. However, the period of refreshing and the sink-change threshold $\mathrm{K}$ have to vary to maximize the lifespan of the whole network by considering the network situation and configuration details.

The simulation results show that the balanced energy consumption regardless of other conditions added to apply multiple sinks. Additionally, while the WiFi enables only 1-hop connections between a router and devices, the proposed scheme uses the multi-hop communication. Thus, even though it uses multiple sinks, it is still cost-effective as the number of gateways can be decreased compared to the original WiFi technology. Also, it is still able to add new sensor nodes in the middle of communication. By periodic refreshing, new sensor nodes can participate in the communication.

However, if the network is small enough to cover with a single sink, the processes for multiple sinks are not necessary. Instead, it can be a burden because of additional calculations. Yet, even though the network does not need other sinks, it still has advantages because it still has scalability.

\section{References}

[1] J. An and W. Chung, "A novel indoor healthcare with time hopping-based visible light communication," in Proc. of 2016 IEEE 3rd World Forum on Internet of Things (WF-IoT), Reston, VA, pp. 19-23, 2016. Article (CrossRef Link)

[2] R. Sarmah, M. Bhuyan and M. H. Bhuyan, "SURE-H: A Secure IoT Enabled Smart Home System," in Proc. of 2019 IEEE 5th World Forum on Internet of Things (WF-IoT), Limerick, Ireland, pp. 59-63, 2019. Article (CrossRef Link)

[3] S. Hwang, J. Jeong and Y. Kang, "SVM-RBM based Predictive Maintenance Scheme for IoT-enabled Smart Factory," in Proc. of 2018 Thirteenth International Conference on Digital Information Management (ICDIM), Berlin, Germany, pp. 162-167, 2018. Article (CrossRef Link)

[4] Intae Ryoo, Kyunghee Sun, Jaesun Lee and Seokhoon Kim, "A 3-dimensional group management MAC scheme for mobile IoT devices in wireless sensor networks," J Ambient Intell Human Comput, 9, 1223-1234, 2018. Article (CrossRef Link)

[5] I. Stanković and W. Dai, "Reconstruction of global Ozone density data using a gradient-descent algorithm," in Proc. of 2016 International Symposium ELMAR, Zadar, pp. 85-88, 2016. Article (CrossRef Link)

[6] H. Asama, "Plenary talk III: Robot \& remote-controlled machine technology for response of disasters and accidents of nuclear power plants," in Proc. of 2012 Proceedings of SICE Annual Conference (SICE), Akita, pp. xii-xii, 2012.

[7] I. Lazar, A. Ghilezan and M. Hnatiuc, "Development of underwater sensor unit for studying marine life," in Proc. of 2016 IEEE 22nd International Symposium for Design and Technology in Electronic Packaging (SIITME), Oradea, pp. 82-85, 2016. Article (CrossRef Link)

[8] S. U. Rehman, S. Berber and A. Swain, "Performance analysis of CSMA/CA algorithm for wireless sensor network," in Proc. of TENCON 2010 - 2010 IEEE Region 10 Conference, Fukuoka, pp. 2012-2017, 2010. Article (CrossRef Link)

[9] HUANG, Pei, et al., "The evolution of MAC protocols in wireless sensor networks: A survey," Communications Surveys \& Tutorials, IEEE, 15.1, 101-120, 2013. Article (CrossRef Link)

[10] Wei Ye, Hohn Heidemann, Devorah Estrin, "An energy-efficient MAC protocol for wireless sensor networks," IEEE INFOCOM, pp. 1567-1576, 2002, Article (CrossRef Link)

[11] Tijs van Dam, Koen Langendoen, "An adaptive energy-efficient MAC protocol for wireless sensor networks," in Proc. of SenSys '03 Proceedings of the 1st international conference on Embedded 
networked sensor systems, pp. 171-180, 2003. Article (CrossRef Link)

[12] Jae-hyen Kim, Seung-Jun Choi, and Ho-nyeon Kim, "Advanced MAC protocol with energy-efficiency for wireless sensor networks," ICOIN, pp. 283-292, 2005. Article (CrossRef Link)

[13] M. Ali, T. Suleman, and Z. A. Uzmi, "MMAC: a mobility-adaptive, collision-free mac protocol for wireless sensor networks," in Proc. of 24th IEEE International Performance, Computing, and Communications Conference, pp. 401 - 407, April 2005. Article (CrossRef Link)

[14] P. P. Czapski, "A Survey: MAC Protocols For Applications Of Wireless Sensor Networks," in Proc. of TENCON 2006 - 2006 IEEE Region 10 Conference, Hong Kong, pp. 1-4, 2006. Article (CrossRef Link)

[15] V. Rajendran, K. Obraczka, J. J. Garcia-Luna-Aceves, "Energy-efficient collision-free medium access control for wireless sensor networks," ACM SenSys, 181-192, 2003.

Article (CrossRef Link)

[16] A. Jhumka and S. Kulkarni, "On the design of mobility-tolerant tdma based media access control (mac) protocol for mobile sensor networks," in Proc. of 4th international conference on Distributed computing and internet technology, vol. 4882, pp. 42-53, 2007.

Article (CrossRef Link)

[17] H. Pham and S. Jha, "An adaptive mobility-aware mac protocol for sensor networks (ms-mac)," in Proc. of IEEE International Conference on Mobile Ad-hoc and Sensor Systems, pp. 558-560, October 2004. Article (CrossRef Link)

[18] Seokhoon Kim, Hangki Joh, Seungjun Choi, Intae Ryoo, "Energy Efficient MAC Scheme for Wireless Sensor Networks with High-Dimensional Data Aggregate," Hindawi Publishing Corporation Mathematical Problems in Engineering, Article ID 803834, 2015.

Article (CrossRef Link)

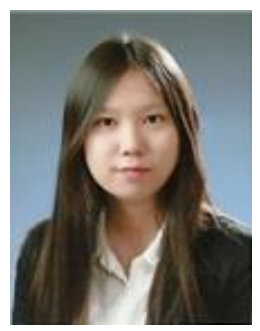

Ahreum Shin received the B.S. degree in computer engineering from Kyung Hee University, Korea, in 2016. She also received M.S. degree in Computer Science \& Engineering in 2018 from Kyung Hee University, Korea.

She has been in Kyung Hee University as a researcher since September 2018. Her research interest includes IoT (Internet of Things) and MAC protocols for wireless sensor networks.

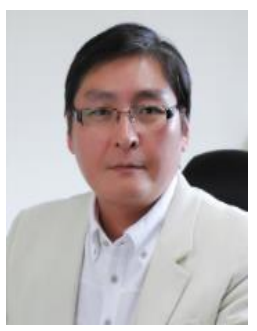

Intae Ryoo received the B.E., M.E. and Doctor Degrees in electronics engineering from Yonsei University, Korea, in 1987, 1989 and 1994, respectively. He received the Ph.D. degree from the University of Tokyo, Japan in 1997. Since 1999, he is a professor of college of electronics and information, Kyung Hee Univ., Korea. His research interests include wireless network system, multimedia communication system, and future Internet.

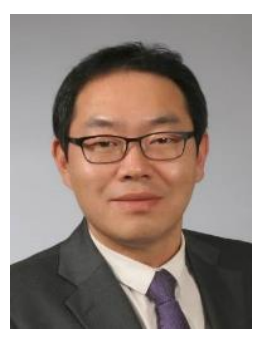

Seokhoon Kim received the B.E. and Ph.D. degrees in computer engineering from Kyunghee University, Korea, in 2000 and 2004, respectively. From 2004 to 2006, he was with IPOne, Inc., Seoul, Korea, where he led various research projects as a Research Engineer. From 2006 to 2009, he was a Research Engineer at Neowave, Inc., Anyang, Korea, where he developed Mobile WiMAX (IEEE 802.16) devices. He was an Assistant Professor in the Department of Mobile Communications Engineering at Changshin University, Changwon, Korea. Since March 2016, he has been with the Department of Computer Software Engineering, Soonchunhyang University, Asan, Korea, where he is currently an Assistant Professor. His research interests comprise Cloud Computing, Internet of Things, Software Defined Networking, Mobile System/Communications, and Machine Learning based on Bigdata. 